\title{
Surgical Safety Checklist: An essential tool to increase patient safety
}

\author{
Pramod Kattel
}

\section{Abstract}

Medical practice without surgery cannot be assumed in the present context. Surgery is the need for individual and safety is his/her right. To increase the safety of the patient by overcoming medical errors, the WHO first introduced the Surgical Safety Checklist more than a decade back. It can be modified as per local needs. Its relevance is seen increasing day by day. When we hear about surgical errors, it is usually felt that it could have been prevented if the checklist were used properly. Teamwork and commitment is a must along with the proper definition of roles of each individual involved in the team. Surgery cannot be considered complete until the safe discharge of the patient is done. Many surgical errors can be prevented by its consistent use. Its use should come as a reflex rather than considering it to be a burden to the existing workload.

Keywords: Checklist; Medical errors; Mortality; Patient safety; WHO.

\section{Author affiliations:}

Department of Obstetrics and Gynaecology, B. P. SMRITI Hospital, Kathmandu, Nepal.

\section{Correspondence:}

Dr. Pramod Kattel,

Department of Obstetrics and Gynaecology, B. P. SMRITI Hospital, Kathmandu, Nepal.

Email: pramodkattel85@gmail.com ORCID: https://orcid.org/0000-0002-64860339

\section{Copyright information:}

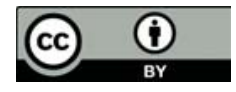

How to cite this article:

Kattel P. Surgical safety checklist: an essential tool to increase patient safety. J Soc Surg Nep. 2020 Dec;23(2):56-58.

DOI:

https://doi.org/10.3126/jssn.v23i2.35841

\section{Introduction}

As a part of global health care, millions of surgeries are performed every year worldwide to save and/or improve life. ${ }^{1,2}$ The same surgeries performed with good aim sometimes cause harm to patients just because of unsafe surgical practice. ${ }^{1}$ About $40 \%$ of hospital adverse events account for surgery. ${ }^{3}$ At least half of the surgical complications that occur are preventable ones. ${ }^{2}$ So there arises a need for good quality surgical services. ${ }^{1}$ To meet the surgical safety demands, the use of a Surgical Safety Checklist is one of them. In addition to it, efforts of decreasing surgical site infection and anaesthesia-related mishaps are also under practice. ${ }^{2}$ The Surgical Safety Checklist was first introduced by the World Health Organization (WHO) in 2007 as a response to increased surgical deaths around the world. ${ }^{4}$ WHO Surgical Safety Checklist is a simple but cost-effective tool to increase patient safety. ${ }^{5}$ The main aim of developing the WHO Surgical Safety Checklist was to decrease errors and adverse events and to increase teamwork and communication in surgery. ${ }^{6}$

The Surgical Safety Checklist comprises a list of activities to be followed before, during, and following surgery. It involves a multi-step process that helps to improve team dynamics, decrease medical errors, and act as back-up to human memory. It involves multidisciplinary teamwork between anaesthesists, surgeons, and nursing staff. ${ }^{4}$ Original WHO Surgical Safety Checklist which comprised of 19 items were revised in 2009 to include few more items making it 22 to be checked in three steps i.e. before induction of anaesthesia-sign in, before skin incision-time out and before the patient leaves the operating room (OR)sign out., ${ }^{2,4}$ 
The components on the list can be altered based on the needs of specialty and type of surgeries e.g. in cases of caesarean section (CS), fetal heart sound should be heard before delivering anaesthesia, and labeling of the baby following delivery should be done along with documentation of time of birth, sex, weight, the status of the child and any gross malformations if noticed. The checklist is prepared to adjust such needs so many modifications on it are found these days. Its importance mostly lies in lengthy surgeries that require many instruments and resources like cotton swabs, gauze pieces, gauze pads, surgical sponges, and towels. The primary objective of following the checklist is to improve the overall safety of the patient. The secondary gain obtained from using it is a decrease in medico-legal litigation risks. The WHO Surgical Checklists standardize safety in delivering care especially in operating theatre (OT) by assessing a series of safety checks., ${ }^{3,5}$ Good communication is an important tool for safer surgical care which is very much required in OT as well. In emergency cases such as "crash caesarean section", the checklist can be used in a modified form. ${ }^{3}$

\section{Objectives}

The objectives of the Surgical Safety Checklists are to deliver safe anaesthesia, to check for adequacy of airway, to assess the risk of blood loss, to take precautions against surgical site infection, to check for the correct site of surgery, to check for any allergies, to maintain accurate and strict swab/instrument counts, to identify specimens, to maintain good communication between whole OR team and to follow surgical outcome. ${ }^{1}$

\section{Components}

There are different components that need to be included in Surgical Safety Checklists which includes patient's identity, name of surgical procedure, surgical site and marking of site (if applicable), consent, past medical and surgical history, required investigations, use of functional Pulse Oximeter, history regarding any known allergy, observation of urine drainage following urinary catheter application, assessment of airway and risk of aspiration and readiness to tackle at time of need, intravenous (IV) access along with IV fluids and readiness of blood/blood products as per need, introduction of team members and definition of role of each, reviewing of patient and surgery including assumption of possible complications and readiness for it, prediction of surgical time, readiness of operative equipments including confirmation of sterility and counts, confirmation of provision of pre-operative antibiotic, displaying of images on display board (if applicable), confirmation of counts of instruments, gauzes and surgical sponges, correct labeling of specimens (if applicable), checking patient pad site of electro-cautery after application and following surgery, assessment regarding recovery of patients and last but not the least includes documentation of all events. ${ }^{2,6}$

\section{Pre-requisites}

The use of the Surgical Safety Checklist requires a commitment by OT staff to increase its compliance. ${ }^{4}$ The operating surgeon should be familiar with the checklist and should advocate to follow it regularly in all institutions. ${ }^{3}$ The leadership of one should be established and the roles of everyone involved in OT should be pre-determined for its consistent practice. Timely meetings regarding its importance, implementation process, improvement following implementation, technical difficulties, and ways to solve it should be done. Health personnel involved should be motivated time and again. Items on the checklist need to be reviewed for practicality and relevance and if needed modification should be done as per local need. Posters of checklist need to be displayed at relevant sites of OT for its widespread application. It is recommended to use board and marker mentioning details of patients, surgical information, and the team involved along with counts of instruments and swabs. The checklist needs to be filed on the medical records of the patient after completion of the task and is signed by an authorized person. ${ }^{5}$

\section{Benefits}

The introduction of the Surgical Checklist enhances the safe delivery of anaesthesia and surgical care. ${ }^{1}$ It encourages checking facts along with the establishment of a good environment ensuring good teamwork. Besides, it helps to decrease the risk of medico-legal litigation to health workers. The implementation of the checklist is to avoid preventable surgical errors which may lead to varied consequences from minor to grave ones. ${ }^{4}$ The application of the checklist will help to improve patient safety by decreasing mortality and complications. ${ }^{3}$ It takes care of error avoidance on part of history and ensures safe delivery of anaesthesia, airway management, identification of the patient, indications of surgery, site of surgery, an examination of a patient before surgery, surgical safety techniques, foreign body retention, and ways to prevent infection. ${ }^{4}$ It is the compilation of teamwork for the overall safety of patients. ${ }^{2,4}$

\section{Challenges}

The compliance for its effective implementation can be affected by factors of limited resources like shortage and rapid turnover of hospital staff. ${ }^{5}$ Use of it may cause duplication of some activities with existing hospital protocols. Sometimes on the course of using the checklist miscommunication between ward and OT staff may occur. Some staff may consider the checklist follow as a burden in addition to the existing workload. ${ }^{4}$ There may be a lack of regular supply of checklist forms for documentation as it is not usually given priority by administrative staff. It demands teamwork which might be considered difficult by some staff. It is commonly observed that some hospital staff feel difficult to accept new things which increase the further challenge on its consistent use. ${ }^{1}$

\section{Solutions}

Though there are many challenges there are ways to decrease them. For this, the staff needs to be motivated to change their behaviour. ${ }^{2}$ There should be avoidance of 
duplication of works. The importance of a checklist on the benefit of patient care should be highlighted. Staff should be counseled regarding their added satisfaction in patient care. Its importance in increasing patient safety, as well as decreased litigation risks of hospital/staff, should be emphasized. Local ownership should be developed and "tried and tested" local checklist should be followed or new development can be done based on local needs. All staff including new ones should be trained for its widespread use even if the turnover rate of staff is high. Effective implementation can be done via direct observation of activities, group education regarding its use, and long-term assistance along with regular assessment, monitoring, and auditing followed by discussion and feedback provision. ${ }^{1}$

\section{References}

1. Ellis R, Nor AIM, Pimentil I, Bitew Z, Moore J. Improving surgical and anaesthesia practice: Review of the use of the WHO safe surgery checklist in Felege Hiwot Referral Hospital, Ethiopia. BMJ Qual Imrpov Rep. 2017;6(1):u207104.w6251.

2. Haynes AB, Weiser TG, Berry WR, Lipsitz SR, Breizat AHS, Dellinger EP et al. A surgical safety checklist to reduce morbidity and mortality in a global population. N Engl J Med. 2009;360:491-99.

3. Singh SS, Mehra N, Hopkins L. Surgical safety checklist in obstetrics and gynaecology. J Obstet Gynaecol Can. 2013 Jan;35(1):82-85.
In addition to the use of a Surgical Safety Checklist, there should be a standard system for instrument sterilization, packing, storing, listing, and recording.

\section{Conclusion}

Patient's benefit and safety are of prime importance in any surgical procedure. To increase the patient's safety, a Surgical Safety Checklist was developed. In the present context, it is useful for the physical safety of patients along with the economic and psychological safety of health care providers by decreasing litigation risks. It can be modified as per local needs. The motivation for its use and consistent practice is a must.

4. Al-Qahtani AS. The surgical safety checklist: Results of implementation in otorhinolaryngology. Oman Med J. 2017 Jan;32(1):27-30.

5. Lilaonitkul M, Kwikiriza A, Ttendo S, Kiwanuka J, Munyarungero E, Walker IA et al. Implementation of the WHO surgical safety checklist and surgical swab and instrument counts at a regional referral hospital in Uganda-a quality improvement project. Anaesthesia. 2015;70(12):1345-55.

6. World Health Organization. WHO surgical safety checklist 2009.2009. 